\title{
THE EFFICACY AND USEFULNESS OF ULTRASOUND- GUIDED TRANSTHORACIC FNAC OF CHEST LESIONS- REVIEW OF 306 CASES
}

\author{
Krishnaveni Nayanagari1, Sarada Bakka², Vasundhara Nagireddy33, Deepa Chinna Polu4 \\ 1 Professor \& HOD, Department of Radiology, Government General Hospital, Ananthapuramu. \\ ${ }^{2}$ Assistant Professor, Department of Radiology, Government General Hospital, Ananthapuramu. \\ ${ }^{3}$ Assistant Professor, Department of Radiology, Government General Hospital, Ananthapuramu. \\ 4 Tutor, Department of Radiology, Government General Hospital, Ananthapuramu.
}

\begin{tabular}{l}
\hline ABSTRACT \\
\hline BACKGROUND \\
Objectives - To assess the effectiveness of this simple, safe, ultrasound guided fine needle aspiration cytology in thoracic masses \\
and correlation of radiological findings with cytological reports. As an initial procedure to differentiate malignant, benign and \\
infective pathology for early diagnosis, cell typing of bronchogenic carcinoma, and to decrease more invasive and expensive \\
procedures considering the poor economic status of people in our area.
\end{tabular}

\section{MATERIALS AND METHODS}

A cross sectional study of 306 cases thoracic lesions conducted in department of Radiology for the period of 3 years. These thoracic lesions are peripheral location and which are located easily on ultrasound. Material was aspirated by using 22 gauge spinal needles under ultrasound guidance sent for cytology. Patient observed for complications for one hour.

\section{RESULTS}

Of these 306 FNAC results $61.76 \%$ were pulmonary malignancies, 13\% infective pathology 5.2\% meditastional lesions, 8.16\% nonspecific and $11.76 \%$ inadequate. Malignancy detection rate is $82 \%$. $0.65 \%$ developed pneumothorax and pulmonary haemorrhage, which did not require any treatment.

\section{CONCLUSION}

Ultrasound guided FNAC using a 22-gauge spinal needle can be used safely as day-care, bed side procedure for diagnosis of thoracic masses and useful to decide the mode of treatment in a shortest possible time and precludes more invasive procedures.

\section{KEYWORDS}

FNAC, Ultrasound, CT, Bronchogenic Carcinoma, Tuberculosis.

HOW TO CITE THIS ARTICLE: Nayanagari K, Bakka S, Nagireddy V, et al. The efficacy and usefulness of ultrasound- guided transthoracic FNAC of chest lesions- review of 306 cases. J. Evolution Med. Dent. Sci. 2017;6(10):790-795, DOI: $10.14260 /$ Jemds/2017/171

\section{BACKGROUND}

Ultrasound (US) guided Fine Needle Aspiration Cytology (FNAC) of thoracic lesions is a well-established, safe, bed side technique. It is widely practiced as an initial diagnostic and supportive procedure for thoracic lesions at present. This method had gone through different modalities like fluoroscopy, ultrasound guided, CT guided and transesophageal to transbronchial procedure. It is a well suited for mediastinal, hilar, pleural, chest-wall and peripheral lung lesions with good acoustic window. Chest wall, pleural and peripheral lung lesions are generally hypoechoic relative to their surrounding tissues. ${ }^{1}$

A time tested procedure for its diagnostic accuracy, feasibility, low complication rate and found to be safe and effective. It can be performed with a basic, two dimensional grey scale ultrasound in hospitals with poor infrastructure. It is a less invasive and can be repeated and the complication

Financial or Other, Competing Interest: None.

Submission 22-12-2016, Peer Review 19-01-2017,

Acceptance 25-01-2017, Published 02-02-2017.

Corresponding Author:

Dr. Krishnaveni Nayanagari,

\#201, Navayuga Gokulum Residency,

Hari Hara Temple Road, Ashok Nagar,

Ananthapuramu-515004.

E-mail:drnkrishnav@gmail.com

DOI: $10.14260 /$ jemds $/ 2017 / 171$ rate is low, ${ }^{2}$ for pulmonologist when sputum is scanty or not available for examination or difficult peripheral location of mass for bronchoscope and clinical status of the patient is not suitable for bronchoscopy, FNAC can be done either by using ultrasound or CT scan. It is an effective procedure for establishing tissue diagnosis of lung neoplasm.

FNAC can be used to differentiate both neoplastic and inflammatory conditions of the masses and resulted in a decrease in the performance of procedures that are more invasive in nature. ${ }^{3}$ Most of the larger lesions and lesions abutting the chest wall are visible through sonography through inter costal spaces. Vascular or other cardiac structures are identified carefully and can be safely avoided by grey scale or applying colour Doppler. Pneumothorax is a common complication. The rate of pneumothoraces incidence reported in the literature varies from $9 \%$ to $24 \% .4$ Several small studies claim that the use of very fine needles and immediate cytological assessment of material helps to decrease procedure repetition and reduce the rate of pneumothorax. ${ }^{5}<5 \%$ of patients complain of minor haemoptysis. Contraindications to FNAC are unconscious, uncooperative patients or in those with respiratory failure, hemorrhagic diathesis, intractable coughing or pulmonary hypertension. 6

The present study was performed to determine the accuracy and complications of US guided FNAC and its ability 
to differentiate benign, malignanant and infective pathologies for early diagnosis and management.

\section{MATERIAL AND METHODS}

Cross sectional study carried out in department of Radiology for the period of 3 years from 2008-2011 under ultrasound guidance. The age groups studied are in between 18 to 85 yrs. The following locations of lesions are taken for the procedures are (Figure 1a,b,c,d) eripheral lung lesions/lesions extending to periphery and pleural lesions, extra pleural lesions. Anterior and posterior mediastinal lesions abutting chest wall, Simultaneous FNAC of mass lesions in other organs for correlation

A short clinical history and list of medications being taken at that time were enquired to prevent complications. Chest Xrays and CT images were made available before aspiration. Written information was given to all patients before the procedure and informed consent was obtained in a written form, from all patients. The patient was positioned according to the anatomical site of lesion; a 22 gauge spinal needle with a central stylet was used. The 20-22 gauge needle is commonly used for good yield. The skin entry site was sterilized with antiseptic; the aspiration needle is then directed towards the lesion during suspended respiration. Presence of the needle tip within the lesion is observed by real time display. The plunger of the syringe is then removed. Continuous suction was then applied while rotating and moving the needle to and fro during suspended respiration. Aspiration was performed.

Aspirated material is directly put on the glass slide, smeared and fixed immediately. The material can also be transported in saline or $50 \%$ alcohol.

Scanning of the anterior mediastinum was done through suprasternal or parasternal approaches. This is performed while the patient is sitting or lying supine. Posterior mediastinal mass lesions were approached posterior paraspinal region with hands extended on to the head.

\section{RESULTS}

306 cases of peripherally situated chest wall, rib, muscle, pleura, lung and mediastinal lesions, underwent US guided FNAC in department of Radiology. Analysis was done, based on malignancy types, location and infective cytology. Simultaneous study of other lesions present in abdomen or any other sites was also done for confirmation of the lesion. FNAC diagnostic accuracy was measured by correlating with clinical picture, radiological impressions, microbiological information, histopathological examination, therapeutic response and metastatic disease elsewhere in the body that were consistent with primary.

Out of 306 cases $70 \%$ are males and $30 \%$ are females.

Among mass lesions when analyzed on FNAC results, pulmonary malignancies predominates the picture $(61.76 \%)$ followed by infective pathology (13\%) and meditational malignancies (3.93\%), inadequate aspiration (11.76\%), and non-specific $(8.16 \%)$ (Table1). Among 306 cases 201 cases are positive for malignancy with the detection rate of $82 \%$.

\begin{tabular}{|c|c|c|}
\hline Pulmonary Malignancies & 189 & $61.76 \%$ \\
\hline Mediastinal Malignancies & 12 & $3.93 \%$ \\
\hline Benign Mediastinal Lesions & 04 & $1.3 \%$ \\
\hline Infective Pathology & 40 & $13 \%$ \\
\hline
\end{tabular}

\begin{tabular}{|c|c|c|}
\hline Non-Specific & 25 & $8.16 \%$ \\
\hline Inadequate Material & 36 & $11.76 \%$ \\
\hline Total & $\mathbf{3 0 6}$ & $\mathbf{1 0 0} \%$ \\
\hline Table 1. General results \\
\hline
\end{tabular}

Lung malignancies are also grouped as small cell and nonsmall cell types, and non-small cell lung carcinoma (NSCLC) constitutes $76 \%$. The most common lung malignancy was squamous cell carcinoma (43.3\%) followed by adenocarcinoma $(17.9 \%)$, poorly differentiated carcinoma (6.8\%) small cell carcinoma (5.8\%), adenosquamous $(2.1 \%)$ and large cell carcinoma (1.5\%). (Table2). Sqamous cell carcinoma is more common among males. Mediastinal lesions are 16 in number and thymomas predominates the lesions. Most of the mediastinal lesions needed biopsy for further evaluation. And role of cytology is accurate to differentiate malignant and infective lesions (Table 3).

In infective pathology $47 \%$ are non-specific infections followed $38 \%$ tuberculosis and $7.5 \%$ of fungal infections.

Overall, $86 \%$ of cytological and radiological concordance observed and $18 \%$ of discordance (Table 4 ) is noted among non-resolving chronic infections mimicking lung masses. In our study complications rate is $0.65 \%$ which is very negligible and complications observed are pneumothorax and haemoptysis.

\begin{tabular}{|c|c|c|}
\hline Diagnosis & Number & Percentage \\
\hline Sqamous Cell Carcinoma & 82 & $43.3 \%$ \\
\hline Adenocarcinoma & 34 & $17.9 \%$ \\
\hline Adenosquamous Carcinoma & 04 & $2.1 \%$ \\
\hline Large Cell Carcinoma & 03 & $1.5 \%$ \\
\hline Small Cell Carcinoma & 11 & $5.8 \%$ \\
\hline Poorly Differentiated Carcinoma & 13 & $6.8 \%$ \\
\hline Metastasis & 09 & $4.1 \%$ \\
\hline $\begin{array}{c}\text { Other Malignancies } \\
\text { (Round Cell and Spindle Cell) }\end{array}$ & 10 & $5.2 \%$ \\
\hline Suggestive of Malignancy & 23 & $12 \%$ \\
\hline Total & 189 & $100 \%$ \\
\hline \multicolumn{3}{|c|}{ Table 2. Pulmonary malignant tumour types } \\
\hline
\end{tabular}



Ant: Anterior; Med: Mediastinal; Post: posterior; mal: Malignant. 


\begin{tabular}{|c|c|c|c|}
\hline $\begin{array}{c}\text { Sl. } \\
\text { No. }\end{array}$ & $\begin{array}{c}\text { Clinical/ } \\
\text { Radiological }\end{array}$ & Cytology & Correlated \\
\hline 1. & Malignancy & $\begin{array}{c}\text { No Malignant } \\
\text { Cells }\end{array}$ & $\begin{array}{c}\text { Sputum positive } \\
\text { for TB }\end{array}$ \\
\hline 2. & Malignancy & - & $\begin{array}{c}\text { Resolved } \\
\text { Completely }\end{array}$ \\
\hline 3. & $\begin{array}{c}\text { Suspected } \\
\text { Mass }\end{array}$ & TB Granuloma & AFB Positive \\
\hline 4. & $\begin{array}{c}\text { Non Resolving } \\
\text { Pneumonia }\end{array}$ & $\begin{array}{c}\text { Inflammatory } \\
\text { Cell }\end{array}$ & Resolved \\
\hline 5. & $\begin{array}{c}\text { Mass with } \\
\text { SVCO }\end{array}$ & TB Granuloma & $\begin{array}{c}\text { TB Granuloma } \\
\text { (Open Biopsy) }\end{array}$ \\
\hline 6. & $\begin{array}{c}\text { Malignancy } \\
\text { Caseating } \\
\text { Necrosis }\end{array}$ & $\begin{array}{c}\text { Chronic } \\
\text { Pathology on } \\
\text { Biopsy }\end{array}$ \\
\hline 7. & $\begin{array}{c}\text { Golden s Sign } \\
\text { Table 4. Discordance in infective pathology } \\
\text { between imaging \& cytology-18\% }\end{array}$ & $\begin{array}{c}\text { Inflammatory } \\
\text { Cells }\end{array}$ & $\begin{array}{c}\text { Inflammatory } \\
\text { (Repeat FNAC) }\end{array}$ \\
\hline \multicolumn{3}{|c|}{}
\end{tabular}

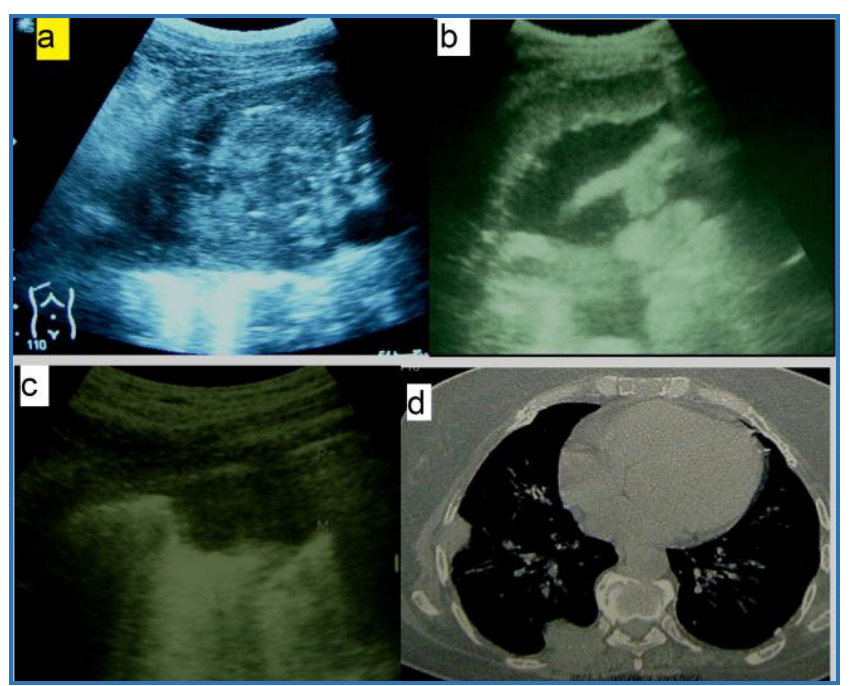

Figure 1. Peripheral Thoracic Masses, a. Large peripheral mass lesion, b. Nodular pleural thickening, c. Small peripheral mass lesion, $d$. Rib lesion with peripheral soft tissue density

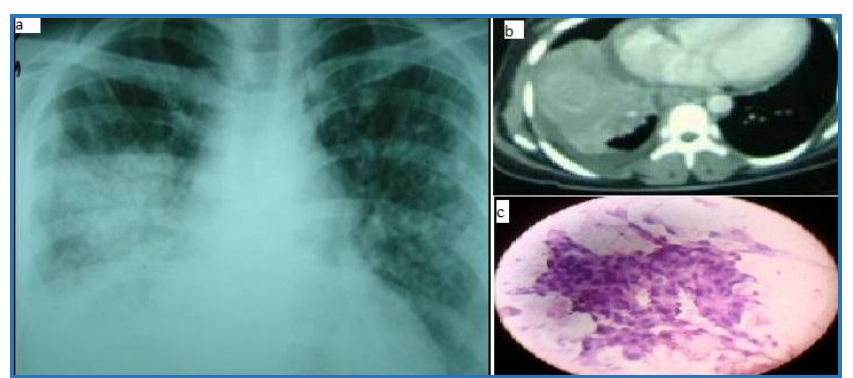

Figure 2. X-Ray and CT chest shows right lower lobe consolidation with minimal effusion and with cytology of hyperchromatic pleomorphic nuclei suggestive of squamous cell carcinoma

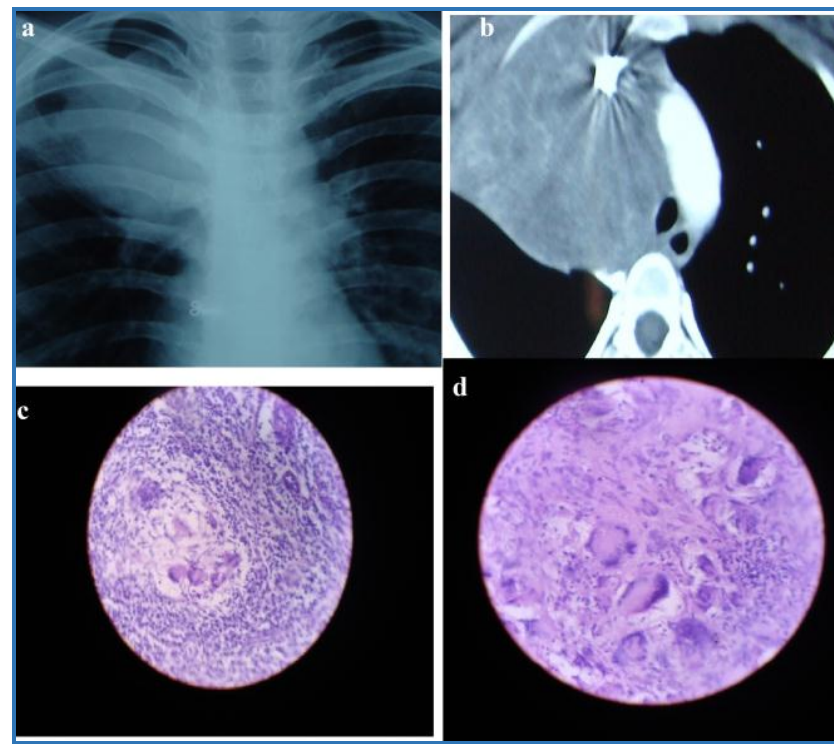

Figure $3 a$ \& b. Plain X-Ray \& CECT chest shows right upper lobe mass with encasement of Superior vena cava and right Pulmonary Artery, $c$ \& d. Langerhan type giant cells with granulomas showing central caseating necrosis surrounded by epitheloidal cells, rimmed by lymphocytes suggestive of tuberculosis



Figure $4 a$ \& b. Plain X-Ray \& CECT chest shows right side osteolytic rib lesion with soft tissue density, c. Cytology smear suggestive of multiple myeloma, $d . X$-ray skull with multiple lytic lesions consisting with multiple myeloma 


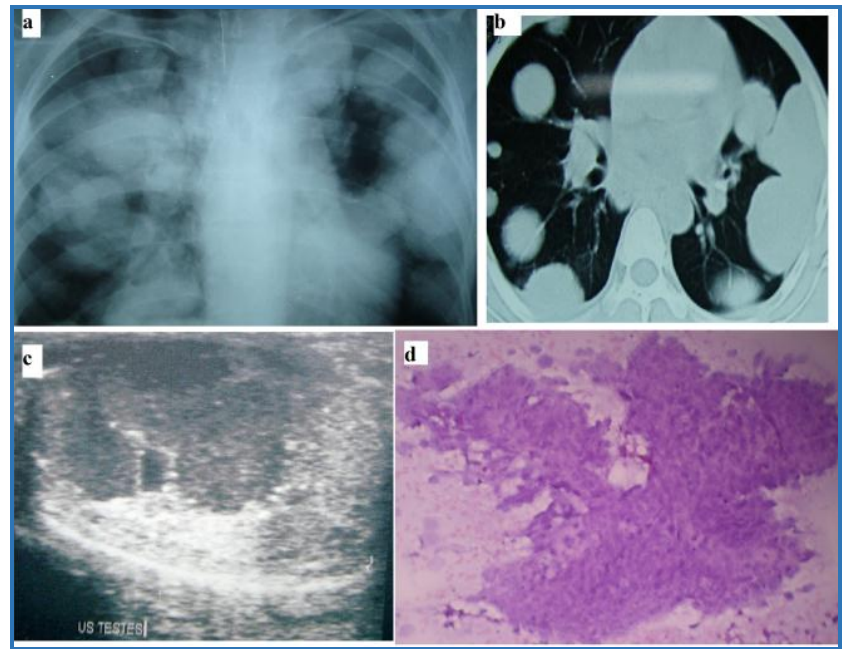

Figure $5 a$ \& b. Plain X-Ray \& CECT chest shows multiple metastatic deposits, c. Ultrasound scrotum shows testicular mass with microlithiasis d. Cytology smear of testicular mass showing poorly differentiating pleomorphic nuclei with mitotic tumour cells suggestive of embryonal cell carcinoma
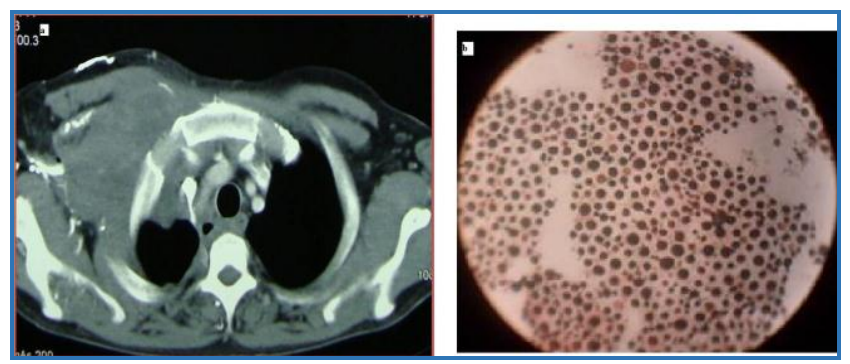

Figure 6a. CT chest shows soft tissue chest wall mass with intrathoracic extension, b. Cytology smear with special stain showing cryptococcosis

\section{DISCUSSION}

Transthoracic FNAC is one of several methods for establishing tissue diagnosis of thoracic lesions, peripheral intra thoracic nodules and lung masses, which are frequently observed on chest ultrasounds and very much approachable for FNAC. The approach of peripherally situated lesions is difficult for bronchoscopy and for sputum cytology and also yield found low for such lesions. US was found as an ideal tool to assist in FNAC/biopsy procedures, with real time demonstration of the position of the needle. It can frequently replace CT guidance at much lower cost. The ultimate goal of any image guided histological or cytological procedure is to reach a definite diagnosis with reasonable accuracy and minimum side effects which precludes invasive, expensive procedures and unnecessary thoracotomy. However the gold standard is the histological diagnosis of the lesion either by biopsy or resection of the lesion. But immediate a provisional diagnosis can be obtained by Cytological evaluation in a shortest possible time.

A final result of FNAC depends on factors like good sampling, location, the nature of the lesion, and amount of aspirated material. Inconclusive FNAC for lung lesions is possible due to inadequate sampling, few cells, abundant necrosis or hemorrhages.

Selection of cases depend mainly on its peripheral location, either in sub pleural region or central mass lesion, extending on to periphery, and anterior, posterior mediastinal mass lesions which are close to chest wall (Figure 1a,b,c,d). This selection will help for good yield and less complications. The presence of bony cage and the surrounding aerated lung, often create problem of localization in ultrasound.

In our study all cases are selected with guidance of x-ray chest or CT image. The size criteria followed is 10 to $20 \mathrm{~mm}$.

In our study the malignancy detection rate is $82 \%$, in literature studies showed the sensitivity range from $89-99 \%$ as per Madan $\mathrm{M}^{\text {et }} \mathrm{al}^{7} \mathrm{~A}$. H. Diacon et al ${ }^{8}$ 95\%, Yun Gong et $\mathrm{al}^{9}$ $85 \%$, with $85 \%$ which is close to our study.

For cases identified as malignant on cytology, attempt was also made to determine histological type and subtypes of primary malignancy. In cases of metastatic deposits the cell type and primary site was also established by simultaneous FNAC of other suspected lesions on ultrasound.

Histological typing can be done as epithelial and nonepithelial malignant lesions. Most thoracic malignancies are epithelial in origin and sometimes FNAC should be initial procedure of choice for evaluation of intra-thoracic lesions. Mesenchymal tumours and lymphomas which has high intercellular adherence requires biopsy of the lesion

Positive result by FNAC is valued; however a negative result does not exclude the presence of disease. Cases with negative result for malignancy will be followed till the lesion is resolved or evaluated with other invasive procedures. It is of note only positive findings provide valuable information and might prevent the need for more invasive diagnostic procedure. (Figure 2). Poorly differentiating carcinomas require immune-histo-chemistry for sub-typing the lesion. Non-specific negative results should be supported further by negative clinical, negative radiological and other findings (Negative for microorganisms). Every case should be considered in a multidisciplinary manner and to avoid false negative results. Benign specific lesion is defined when radiological, clinical impression is compatible with pathological findings like granuloma, fungal or mycobacterium infection, organising pneumonia and benign tumours. Benign non-specific or inadequate smear contains normal parenchyma, fibrosis, debris or nonspecific inflammation. Clinical, radiological benign lesions with benign cytology require follow up and no immediate surgical intervention required. Lesion with benign findings on FNAC, but clinical and radiological evidence suggesting of malignancy warrants and requires closed or open biopsy or surgical excision (Figure 3a,b,c,d).

Bronchogenic carcinomas are the most common pulmonary malignancies observed in our study. Two broad classes are distinguished as non-small cell lung carcinoma (NSCLC) and small cell lung carcinoma (SCLC). Among nonsmall cell type squamous cell carcinoma predominates in our study $(43.3 \%)$, correlating with studies of shah et al ${ }^{10}$ and Saha A et al ${ }^{11}$ where squamous cell type incidence $45 \%$, $42.6 \%$ respectively.

This neoplasm is most common in men and it is closely related to smoking. The incidence of squamous cell type was more, possibly due to more number of male patients included in our study.

$40 \%$ of lung cancers are adenocarcinoma, which usually originates in peripheral lung tissue. $17.9 \%$ adenocarcinoma 
were observed in our study and followed by adenosquamous $(2.1 \%)$ which is a type of combination of different cell types.

The other group, small cell carcinomas, the cells contain dense neurosecretory granules, 12 and most cases arise in the larger airways. 60 to 70 percent have extensive disease by the time of their presentation, posing difficulty for radiological diagnosis, due to its infiltration into surrounding planes.

$14 \%$ of discordance noted in radiological impression and cytology reports of malignancy. It is observed that lesions of infective pathology and non-specific pneumonia mimics malignancy. FNAC will be the initial procedure of evaluation in such lesions. In one case of mass lesion with air fluid levels due to necrosis, FNAC concluded this as squamous cell carcinoma Typical and atypical presentations of bronchogenic carcinoma mimicking, consolidation observed in our study mainly in younger age group (Figure 2),

$4.1 \%$ thoracic lesions of our study are metastatic in origin. To establish the primary site of origin, cytomorphology of secondary deposit, correlated and interpreted with the clinical context and radiological evaluation or FNAC of other sites affected.

One female patient presented with chest wall swelling with osteolytic lesion of ribs with soft tissue on chest radiograph. FNAC revealed multiple myeloma of the rib. It precluded further invasive procedures by simultaneous examination of other areas (skull) demonstrating multiple osteolytic lesions matching with rib cytology (Figure 4a,b,c,d).

One young male patient presented with multiple soft tissue in chest radiograph suggesting of multiple metastatic disease. He underwent ultrasound abdomen for the evaluation of primary disease which revealed bilateral testicular microlithiasis and non-homogenous mass lesion in one testes with multiple Para aortic nodes. FNAC performed from chest lesion and Para aortic nodes and testes and turned out as non seminomatous tumor testes with secondaries lung and abdomen (Figure 5a,b,c,d).

Non-resolving and chronic pulmonary infections like pulmonary tuberculosis are always a source of confusion when sputum results are not available and pose difficulty in diagnosis. We observed $38 \%$ of cases of TB among infective group. Our study reaffirms the use of FNAC in one case of pulmonary tuberculosis mimicking malignancy, by encasing greater vessels and causing superior veno caval obstruction. Though initial FNAC revealed granulomatous reaction and due to high clinical and radiological suspicion of malignancy patient was subjected for open biopsy, which finally revealed mediastinal and pulmonary tuberculosis. Among infective pathology of our study, clearly TB is an important differential diagnosis of a mass mimicking malignancy particularly in Asia.

FNAC will be complemented by ancillary techniques such as immunocytochemistry, electron microscopy, mucin stains and microbiological stains.13 In our study two cases with microbiological stains helped to differentiate malignancy from infective nature and helped for final diagnosis of cryptococcosis (Figure 6a,b) and mucormycosis. In our study discordance in infective pathology between imaging \& cytology $18 \%$. (Table 4 ).

The diagnosis of mediastinal lesions can be challenging because of the numerous variable benign and malignant processes occurring at this site. FNAC is commonly used to sample tumours in all compartments of the mediastinum, especially for evidence of metastatic disease. However In mediastinal lesions the clinical and radiological findings may not help for a definitive diagnosis and a histological diagnosis is often required. FNAC can be used as initial technique and can be verified by histopathological examination for further evaluation. In contrast to pulmonary and pleural lesions mediastinal mass lesions are with adherence cellularity, and is difficult to obtain in FNAC which is commonly observed in lymphomas. Technique depends on the localization of the lesion, clinical factors, and the availability infrastructure. ${ }^{14}$

The most common causes of an anterior mediastinal masses are: thymoma, teratoma, thyroid disease and lymphoma. Masses of the middle mediastinum are congenital cysts, including foregut and pericardial cysts, while those arising in the posterior mediastinum are often neurogenic tumours.

In our study 16 cases of mediastinal lesions observed and more common site is anterior mediastinum. 5 cases of thymomas, 2 cases of lymphomas were diagnosed on FNAC. It is primary modality in the work up of suspected thymomas. Sub typing thymomas, on cytology is not valuable and if a pathologist is able to confidently issue a diagnosis of thymomas on FNAC, this information should suffice to direct management. 15

Teratoma which are common in anterior mediastinum, cytology showed pleomorphic cells or non-specific cytology in our study, needed surgical exploration. One case of mediastinal cyst which showed inflammatory smear was completely resolved on follow up. One case of benign schwannoma which was provisionally diagnosed on radiological findings as neurogenic tumour in posterior mediastinum, showed no malignant cells on cytology.

Among complications of FNAC, the prevalence of pneumothorax and haemoptysis studied in relation to the depth and size of the lesion proved to be directly related. ${ }^{16}$

Selecting larger mass lesions and lesions extending and abutting the chest wall with no intervening parenchyma and which are easily visible on ultrasound will reduce complications. Application of colour flow mapping to identify vascular or other cardiac structures prevents haemoptysis a grave complication. Several small studies in literature claim that the use of very fine needles and immediate cytological assessment of material reduces the number of attempts and reduced the rate of pneumothorax ${ }^{17}$ and other complications like pneumo-mediastinum, air embolism and haemothorax are extremely rare after FNAC of Lung.

Less than $5 \%$ of patients complain of minor haemoptysis. In our study the incidence is $0.65 \%$ which is very less possibly due to utilizing x-ray and CT films before doing the procedure. In one case of pneumothorax which developed during the procedure was immediately identified on ultrasound itself and confirmed on x-ray chest and timely management was given to save the patient. One case presented with minimal haemoptysis and needed no treatment.

\section{CONCLUSION}

Transthoracic FNAC is a valuable method that can be used as initial procedure for evaluation of peripheral or visible intrathoracic lesions. 


\section{Limitations}

Ultrasound has limitations for deep seated and small lesions where CT guidance is the safe and preferred technique in FNAC/ BIOPSY of intra-thoracic lesions. Although USG guidance can be tried in superficial lesions or in situation where CT scan is not available.

It is a day care method which can be practiced on bedside. When compared with bronchoscopy, thoracotomy and open lung biopsy it is cost effective and simple with good diagnostic yield. Results can be obtained in short period and helps for timely appropriate management.

\author{
Abbreviations \\ FNAC- Fine Needle Aspiration Cytology. \\ CT- Computed tomography. \\ NSCLC- Non Small Cell Lung Carcinoma. \\ SCLC- Small Cell Lung Carcinoma. \\ TB- Tuberculosis.
}

\section{REFERENCES}

[1] Yang PC. Ultrasound guided transthoracic biopsy of peripheral lung, pleural and chest wall lesions. J Thorac Imaging 1997;12(4):272-84.

[2] Diacon AH, Schuurmans MM, Theron J, et al. Safety and yield of ultrasound assisted transthoracic biopsy performed pulmonologists. Respiration 2004;71(5):519-22.

[3] Silverman JF. Inflammatory and neoplastic processes of the lung: differential diagnosis and pitfalls in FNA biopsies. Diagn Cytopathol 1995;13(5):448-62.

[4] Tan KB, Thamboo TP, Wang SC, et al. Audit of transthoracic fine needle aspiration of the lung: cytological subclassification of bronchogenic carcinomas and diagnosis of tuberculosis. Singapore Med J 2002;43(11):570-5.

[5] Johnsrude IS, Silverman JF, Weaver MD, et al. Rapid cytology to decrease pneumothorax incidence after percutaneous biopsy. AJR Am J Roentqenol 1985;144(4):793-4.

[6] Weisbrod GL. Transthoracic percutaneous lung biopsy. Radiol Clin North Am 1990;28(3):647-55.
[7] Madan M, Bannur H. Evaluation of FNAC in the diagnosis of lung lesions. Turk J Pathol 2010;26(1):16.

[8] Diacon AH, Theron J, Schubert P, et al. Ultrasoundassisted transthoracic biopsy: fine-needle aspiration or cutting-needle biopsy? Eur Respir J 2007;29(2):357-62.

[9] Gong Y, Sneige N, Guo M, et al. Transthoracic fineneedle aspiration vs concurrent core needle biopsy in diagnosis of intrathoracic lesions a retrospective comparison of diagnostic accuracy. Am J Clin Pathol 2006;125(3):438-44.

[10] Shah S, Shukla K, Patel P. Role of fine needle aspiration cytology in diagnosis of lung tumours-a study of 100 cases. Indian J Patho Microbial 2007;50(1):56-8.

[11] Saha A, Kumar K, Choudhuri MK, et al. Computed tomography-guided fine needle aspiration cytology of thoracic mass lesions: a study of 57 cases. J Cytol 2009;26(2):55-9.

[12] Rosti G, Bevilacqua G, Bidoli P, et al. Small cell lung cancer. Annals Oncology 2006;17(Suppl 2):5-10.

[13] O Reilly PE, Brueckner J, Silverman JF. Value of ancillary studies in fine needle aspiration cytology of lung. Acta Cytol 1994;38(2):144-50.

[14] Gonzalez M, Ris HB, Krueger T, et al. Management of anterior mediastinal masses in adult's. Rev Mal Respir 2012;29(2):138-48.

[15] Ali SZ, Erozan YS. Thymoma. Cytopathologic features and differential diagnosis on fine needle aspiration. Acta Cytol 1998;42(4):845-54.

[16] Begum SMKN, Ekram ARMS, Uddin D, et al. Diagnostic accuracy of ultrasound versus computed tomographic guided fine needle aspiration cytology. AKMMC J 2010;1(1):09-14.

[17] Johnsrude IS, Silverman JF, Weaver MD, et al. Rapid cytology to decrease pneumothorax incidence after percutaneous biopsy. AJR Am J Roentqenol 1985;144(4):793-4. 